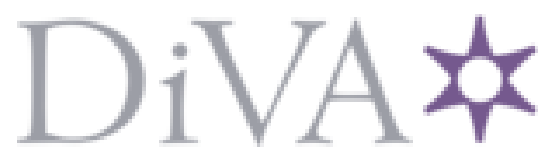

http://www.diva-portal.org

This is the published version of a paper published in Physical Review A. Atomic, Molecular, and Optical Physics.

Citation for the original published paper (version of record):

Jääskeläinen, M., Meystre, P. (2005)

Dynamics of Bose-Einstein condensates in double-well potentials.

Physical Review A. Atomic, Molecular, and Optical Physics, 7 1(4): 043603

http://dx.doi.org/10.1103/PhysRevA.71.043603

Access to the published version may require subscription.

N.B. When citing this work, cite the original published paper.

Permanent link to this version:

http://urn.kb.se/resolve?urn=urn:nbn:se:du-11211 


\title{
Dynamics of Bose-Einstein condensates in double-well potentials
}

\author{
M. Jääskeläinen and P. Meystre \\ Optical Sciences Center, The University of Arizona, Tucson, Arizona 85721, USA
}

(Received 2 December 2004; published 11 April 2005)

\begin{abstract}
We study the dynamics of Bose-Einstein condensates in symmetric double-well potentials following a sudden change of the potential from the Mott-insulator to the superfluid regime. We introduce a continuum approximation that maps that problem onto the wave-packet dynamics of a particle in an anharmonic effective potential. For repulsive two-body interactions the visibility of interference fringes that result from the superposition of the two condensates following a stage of ballistic expansion exhibits a collapse of coherent oscillations onto a background value whose magnitude depends on the amount of squeezing of the initial state. Strong attractive interactions are found to stabilize the relative number dynamics. We visualize the dynamics of the system in phase space using a quasiprobability distribution that allows for an intuitive interpretation of the various types of dynamics.
\end{abstract}

DOI: $10.1103 /$ PhysRevA.71.043603

PACS number(s): 03.75.Be, 03.65.Ge, 05.60.Gg

\section{INTRODUCTION}

Atom optics [1] and the physics of ultracold matter waves have witnessed rapid progress in recent years, due in large part to the experimental achievement of Bose-Einstein condensation [2]. In particular, there has been much recent interest in ultracold atoms in optical lattices, partly because of prospects for quantum computation [3], but also because of the possibility to study fundamental many-body physics in model systems with highly controllable parameters. In an application more closely related to quantum optics, the first experimental realization of a number-squeezed state of the matter-wave field was performed using an optical lattice [4], with the concomitant promise of increased accuracy in interferometry $[5,6]$. The saturation in the amount of squeezing observed at high lattice depths in these experiments was recently explained by the nonadiabaticity of the splitting [7]. Finally, we mention the transition between a Mott-insulating and a superfluid state predicted in Refs. [8,9] and experimentally realized by Greiner and co-workers [10].

A two-well system is the simplest many-body lattice model that exhibits the effects of the competition between two-body interactions and quantum tunneling. This makes it a good candidate for understanding and visualizing the basic physics behind the more complex behavior of many-well systems. Many of the static and dynamical properties of condensates in two-well potentials, such as squeezing [11,12] and the self-trapping of Josephson oscillations $[13,14]$ have been studied. The collapse and revival dynamics [15] of atomic two-mode systems was discussed by Milburn et al. [16], and its extension to optical lattices was studied in Ref. [17]. It was recently suggested as a means to carry out Heisenberg-limited calibration of interferometers [18]. Kalosakas and co-workers have studied the stationary states [19] and have given perturbative estimates [20] of the classical oscillation period and revival time of the so-called boson-Hubbard dimer, or discrete nonlinear Schrödinger dimer, in the weak tunneling limit.

The goal of the present paper is to study the dynamics of ultracold bosonic atomic vapors in time-dependent doublewell potentials. Two limiting cases are of particular interest, the adiabatic limit and the sudden limit. In the first case, atoms initially in the many-body ground state of the doublewell potential adiabatically follow the instantaneous ground state. This regime is of particular interest for the preparation of specific many-body states [4,5]. On the other hand, rapid perturbations of a system initially in its ground state induce nontrivial dynamics that can be used as a probe the properties of the system. For example, certain aspects of the dynamics following the change of the potential depth from a regime where the ground state is superfluid to the Mottinsulator regime and back were recently investigated in Ref. [21], and Ref. [22] studied the nonadiabatic loading of a condensate into an optical lattice and the resulting excitation of collective modes, as experimentally realized in Ref. [23]. Also noteworthy is Ref. [24], which described the evolution of the phase coherence after the atomic system, initially prepared in a Mott-insulating state, was allowed to evolve after a sudden change in parameters placed it in a regime with a superfluid ground state, using in particular a stochastic Gross-Pitaevskii approach. The behavior around the transition was further studied in Ref. [25], which predicts macroscopic oscillations of the order parameter between the superfluid and Mott phases. Finally, Ref. [26] compared the exact quantum dynamics for few atoms with Hartree-Fock-type approximations for the case of nonadiabatic loading of a condensate into a lattice. We note that much of the past work has concentrated on the case of repulsive interactions, partly because for negative scattering lengths the stabilization of the condensate relies on the trap potential counteracting the collapse brought about by the attractive interaction $[27,28]$. This can only be achieved for small condensates, the critical number of atoms depending on the trap geometry [29] and being largest for pancake shapes. The condensate sizes achievable in double-well traps with well-separated minima have been shown to be essentially identical to those for single-well traps [30].

With these previous results in mind, the goal of the present paper is to study the dynamics of Bose-Einstein condensates following sudden changes in the double-well potentials in which they are trapped. Our analysis relies largely on numerical wave-packet dynamics simulations and makes ex- 
tensive use of a Bloch-sphere quasiprobability representation to achieve a simple geometrical interpretation of our results. As such, this study complements our previous paper [5], which concentrates on approximately adiabatic changes of the system parameters.

The paper is organized as follows. Section II discusses our model and establishes the notation. We introduce an approximate mapping of the problem onto an effective onedimensional Schrödinger equation with a quartic effective potential that can be analyzed by the techniques of wavepacket dynamics. Section III presents explicit numerical results of this analysis, and interprets them both in the wavepacket picture and in a phase-space representation on a Bloch sphere. Finally, Sec. IV is a summary and outlook.

\section{MODEL}

In the two-mode approximation, the Hamiltonian of a Bose-Einstein condensate in a double-well trap can be written as [5]

$$
\hat{H}=\frac{\Delta E(t)}{2}\left[\hat{a}_{L}^{\dagger} \hat{a}_{R}+\hat{a}_{R}^{\dagger} \hat{a}_{L}\right]+g(t)\left[\hat{a}_{L}^{\dagger 2} \hat{a}_{L}^{2}+\hat{a}_{R}^{\dagger 2} \hat{a}_{R}^{2}\right],
$$

where $\hat{a}_{L(R)}$ are annihilation operators for particles in the left (right) well, and we have introduced the tunneling energy $\Delta E(t)$ and two-body interaction energy $g(t)$, both of which can be externally controlled and are therefore taken to be functions of time. This two-mode problem is conveniently reexpressed in the Schwinger angular momentum representation of bosonic operators [16,31]. Introducing the angular momentum operators

$$
\begin{gathered}
\hat{J}_{z}=\frac{1}{2}\left(\hat{a}_{L}^{\dagger} \hat{a}_{L}-\hat{a}_{R}^{\dagger} \hat{a}_{R}\right), \\
\hat{J}_{+}=\hat{a}_{L}^{\dagger} \hat{a}_{R}, \\
\hat{J}_{-}=\hat{a}_{R}^{\dagger} \hat{a}_{L},
\end{gathered}
$$

where $\hat{J}_{z}$ is the projection onto a fictitious quantization axis, and the operators $\hat{J}_{ \pm}$are raising and lowering operators along this axis, we can express the state of the matter-wave field in terms of eigenstates $|J, m\rangle$ of the operators $\hat{J}^{2}$ and $\hat{J}_{z}$, where

$$
\hat{J}^{2}=\hat{J}_{x}^{2}+\hat{J}_{y}^{2}+\hat{J}_{z}^{2},
$$

with

$$
\begin{gathered}
\hat{J}^{2}|J, m\rangle=\hbar^{2} J(J+1)|J, m\rangle, \\
\hat{J}_{z}|J, m\rangle=\hbar m|J, m\rangle=\frac{1}{2}\left(n_{L}-n_{R}\right)|J, m\rangle,
\end{gathered}
$$

$J=N / 2, m=-J,-J+1, \ldots, J$, and

$$
\begin{aligned}
& \hat{J}_{+}|J, m\rangle=\sqrt{(J+m+1)(J-m)}|J, m+1\rangle, \\
& \hat{J}_{-}|J, m\rangle=\sqrt{(J-m+1)(J+m)}|J, m-1\rangle .
\end{aligned}
$$

In the angular momentum representation the Hamiltonian (1) reads, apart from a constant function of the total angular momentum eigenvalue $J=N / 2$,

$$
\hat{H}=2 g(t) \hat{J}_{z}^{2}+\Delta E(t) \hat{J}_{x},
$$

where

$$
\hat{J}_{x}=\frac{1}{2}\left(\hat{J}_{+}+\hat{J}_{-}\right) .
$$

The Hamiltonian (9) is the single-axis twisting Hamiltonian familiar from the theory of spin squeezing [32]. We also recall that in the context of atom optics the operator $\hat{J}_{x}$ plays a central role: assuming that the spatial mode functions of the double well are identical and symmetric [5] its expectation value gives the visibility of interference fringes for a condensate released from the trap [5] as

$$
V=\frac{\left\langle G_{1}(0, t, 0)\right\rangle-\left\langle G_{1}(0, t, \pi)\right\rangle}{\left\langle G_{1}(0, t, 0)\right\rangle+\left\langle G_{1}(0, t, \pi)\right\rangle}=\frac{\left|\left\langle\hat{J}_{x}\right\rangle\right|}{J},
$$

where $\left\langle G_{1}(x, t, \theta)\right\rangle$ is the density of atoms at a spatial location for a double-well condensate with a relative average phase $\theta$ between the halves. For these reasons, the Schwinger angular momentum representation is particularly appropriate to discuss the problem at hand.

We proceed by expanding the state $|\psi\rangle$ of the condensate on the $|J, m\rangle$ basis as

$$
|\psi\rangle=\sum_{m=-J}^{J}(-1)^{m} c(m, t)|J, m\rangle,
$$

where the phase factors $(-1)^{m}$ are introduced for convenience, motivated by the analytical form of the eigenstates known for few particles [33]. Alternatively, $|\psi\rangle$ can be expanded in the eigenfunctions $\left|\varphi_{n}(t)\right\rangle$ of the time-dependent Hamiltonian $H(t)$ as

$$
|\psi\rangle=\sum_{n=0}^{N} D_{n}(t)\left|\varphi_{n}(t)\right\rangle,
$$

the eigenfunctions $\left|\varphi_{n}(t)\right\rangle$ being in turn expanded in terms of the states $|J, m\rangle$ as

$$
\left|\varphi_{n}(t)\right\rangle=\sum_{m=-J}^{J} c_{m}^{n}(t)|J, m\rangle .
$$

If the time evolution of the Hamiltonian is sufficiently slow, the dynamics of the condensate follows its change adiabatically [5], the amplitudes $D_{n}(t)$ having then constant magnitudes. In the present work we consider the other extreme of sudden change in the Hamiltonian.

To gain further insight into both the stationary states and the dynamics of the condensate under the Hamiltonian (9), we proceed in a manner similar to the approach of Ref. [12], assuming that for large enough particle numbers the coefficients $c(m, t)$ can be treated as continuous functions of the magnetic quantum number $m$. This leads as we shall see to a formulation of the problem in terms of wave-packet dynamics, an approach that been exploited both in configuration 
space [35] and in phase space [36] and has witnessed much success due in part to its intuitive appeal.

Assuming that the function $c(m, t)$ is sufficiently smooth, we expand it around an arbitrary point $m$ as

$$
c(m \pm 1, t)=\left(c(m, t) \pm \frac{\partial c(m, t)}{\partial m}+\frac{1}{2} \frac{\partial^{2} c(m, t)}{\partial m^{2}}\right) .
$$

This approximation is expected to hold provided that the number of atoms is not too small. Inserting Eq. (15) into the Hamiltonian (9) and expanding the matrix elements (7) and (8) of the operators $\hat{J}_{ \pm}$in powers of $m / J$, we arrive at

$$
\begin{aligned}
\langle m|\hat{H}| \psi\rangle= & \left\{-\Delta E\left(J+\frac{1}{2}\right) \frac{\partial^{2} c(m, t)}{\partial m^{2}}+\left[-\Delta E\left(J+\frac{1}{2}\right)\right.\right. \\
& \left.\left.+\left(2 g-\frac{\Delta E}{2 J}\right) m^{2}+\frac{1}{8 J^{3}} m^{4}\right] c(m, t)\right\} .
\end{aligned}
$$

The continuum approximation implicitly assumes that the number of nonzero amplitudes is not small, an approximation that breaks down close to the crossover to the Mott regime, where a single probability amplitude becomes dominant. It also suffers a breakdown for large attractive interactions when the ground state is dominated by the two states $m= \pm 1$.

Introducing the dimensionless variables

$$
x \equiv \frac{m}{J}, \quad \tau \equiv J \Delta E t
$$

and the dimensionless "energy ratio"

$$
G(\tau) \equiv \frac{4 J g}{\Delta E},
$$

the time-dependent Schrödinger equation results finally in a one-dimensional Schrödinger equation in the (quasicontinuous) variable $x$,

$$
i \frac{\partial c(x, \tau)}{\partial \tau}=-\frac{1}{2 M_{\mathrm{eff}}} \frac{\partial^{2} c(x, \tau)}{\partial x^{2}}+V_{\mathrm{eff}}(x) c(x, \tau),
$$

where the effective mass $M_{\text {eff }}$ is

$$
M_{\text {eff }}=\frac{J^{2}}{2}=\frac{N^{2}}{8}
$$

and the effective potential $V_{\text {eff }}(x)$ is given by the quarticquadratic form

$$
V_{\mathrm{eff}}(x)=[G+1] \frac{x^{2}}{2}+\frac{x^{4}}{8},
$$

where we without loss of any generality have dropped a constant term. The quantum dynamics of the Hamiltonian (9) can thus equivalently be analyzed using one-dimensional wave-packet dynamics in a polynomial potential. In this picture the momentum states correspond to the relative phase states [34]. For repulsive interactions $G>0$, the low-energy eigenstates are approximately harmonic oscillator eigenfunctions, as discussed in Ref. [12]. For attractive interactions, on the other hand, the effective potential changes from having a single minimum at $x=0$ for $G>-1$ to exhibiting a double well for $G<-1$.

For the dynamics we consider specifically the situation where the energy ratio $G$ is suddenly switched from some initial value to a new value $G(\tau>0) \equiv G$ at $\tau=0$, corresponding to a new time-independent effective potential $V_{\text {eff }}(x)$ with eigenstates and eigenenergies $C_{n}(x)$ and $E_{n}$, respectively. In complete analogy with one-dimensional, single-particle quantum mechanics, the dynamics of the "wave function" $c(x, \tau)$ is then given by

$$
c(x, \tau)=\sum_{n=0}^{N} d_{n}(0) \exp \left(-i E_{n} \tau\right) C_{n}(x),
$$

where $d_{n}(0)$ are the projections of $c(x, \tau=0)$ onto the eigenfunctions $C_{n}(x)$. Apart from the factor $(-1)^{m}$, the stationary states of the two-mode condensate are thus approximated by the eigenstates of $V_{\text {eff }}$.

The wave-packet dynamics are conveniently described using the intuitively appealing pictures provided by phasespace representations [36]. For the problem at hand, which is amenable as we have seen to an angular momentum representation, a natural choice is the quasiprobability density on the Bloch sphere $Q(\varphi, \theta)$,

$$
Q(\varphi, \theta)=|\langle\psi \mid \varphi, \theta\rangle|^{2},
$$

where the angular Bloch states are given by [37]

$$
\begin{aligned}
|\varphi, \theta\rangle= & \sum_{m=-J}^{J} \sqrt{\left(\begin{array}{c}
2 J \\
m+J
\end{array}\right)} \sin ^{J+m}(\theta / 2) \cos ^{J-m}(\theta / 2) \\
& \times \exp [-i(J+m) \varphi]|J, m\rangle .
\end{aligned}
$$

This representation allows one to follow the quantum dynamics as the flow of a continuous function on a Bloch sphere.

\section{RESULTS}

With this formal development at hand, we now turn to a discussion of the main results of our numerical study. To set the stage, it is useful to first consider the expansion of the eigenstates $\left|\varphi_{n}\right\rangle$ of the Hamiltonian (1) in terms of the basis states $|J, m\rangle$ [see Eq. (14)]. Figure 1 shows the corresponding probability amplitudes $c_{m}^{0}$ for the ground state $n=0$ of $\hat{H}$ for $G=-2,0,10$, together with the corresponding effective potentials $V_{\text {eff }}(x)$. As the two-body interaction becomes increasingly attractive $V_{\text {eff }}$ undergoes a transition from being a single to a double-well potential. This transition is analogous to the Landau-Ginzburg phase transition familiar from magnetism, laser theory, and other areas of statistical physics. As a result, the projection of the ground state on the magnetic quantum numbers $m$ (or equivalently the population difference $\left.n_{L}-n_{R}\right)$ can be either squeezed or split into a doublepeaked distribution. Specifically, for large repulsive interactions the ground state is highly squeezed [32] in relative number, becoming approximately a Fock state for $G>N^{2}$. This is the double-well analog of the Mott-insulator state $[8,9,38]$ in a periodic lattice. The number squeezing de- 


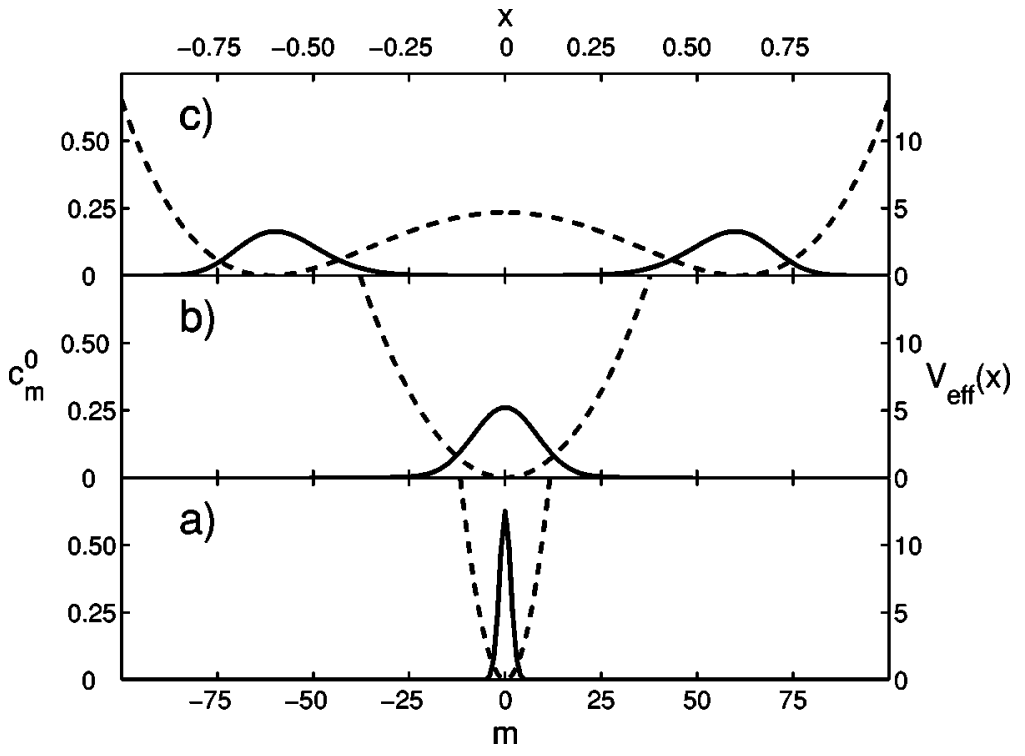

FIG. 1. Probability amplitudes $c_{m}^{0}$ for the ground state of the exact Hamiltonian (9) (solid lines), and effective potential $V_{\text {eff }}(x)$ (dashed lines), $G=$ (a) -2.5 , (b) 0 , and (c) 10 . The distribution of exact ground-state probability amplitudes is consistent with what could be expected for the effective potential.

creases for weaker interactions and in the vicinity of the noninteracting limit the ground state is essentially a coherent state in the relative number distribution with uncertainty $\Delta m \approx \sqrt{N}$. This is essentially a superfluid state. As the interaction becomes attractive the distribution of magnetic quantum states further broadens until $G=-1$, at which point the quadratic term in the effective potential goes to zero and the distribution splits. For large attractive interactions the ground state is essentially a macroscopic superposition (Schrödinger catstate) of two states corresponding to all atoms being in either one well or the other.

Figure 2 shows a gray-scale rendition of the projections $c_{m}^{n}$ of all eigenstates $\left|\varphi_{n}\right\rangle$ of $H$ on the magnetic eigenstates $m$. The darker the shade of gray, the larger $c_{m}^{n}$. The ground-state projections of Fig. 1 correspond to the horizontal line $n=0$, showing again the transition from a squeezed to a doublepeaked distribution as the interaction becomes increasingly attractive. Note the symmetry between the cases of repulsive and attractive interactions, in the sense that the probability amplitudes for eigenstates of high and low quantum numbers $n$ are interchanged under a change of sign of $G$. For large attractive interactions the central part of the double-well potential can be described to a good approximation by a parabolic barrier. Such a potential exhibits scattering resonances that are turned into stationary states by imposing box quantization conditions. It follows that some of the excited states are localized near the top of the barrier [39].

In contrast to Ref. [5], which considers approximately adiabatic changes in the potential double-well potential as a means to prepare specific states of the matter-wave field, we now analyze the response of the atoms to sudden changes in $V_{\text {eff }}$.

The time scale over which the Hamiltonian changes cannot be arbitrarily short since the system has to be adiabatic with respect to the external mode functions of the confining potential. It is known [16] that the two-mode approximation is valid provided that the interaction energy $\mathrm{Ng}$ remains small compared to the energy separation $\hbar \omega$ between trap levels. In addition, the spatial mode functions of particles localized on each side of the double well are well described by shifted Gaussians when the interwell tunneling energy $\Delta E$ is much smaller than their frequencies, which are also of order $\hbar \omega$ [40]. If these two conditions are met, it is easy to modify the energy ratio $G$ in a time $1 / \omega \ll T \ll \hbar / \Delta E, \hbar / g N$ such that the change is adiabatic with respect to the double well, but practically instantaneous as far as the many-body dynamics is concerned.

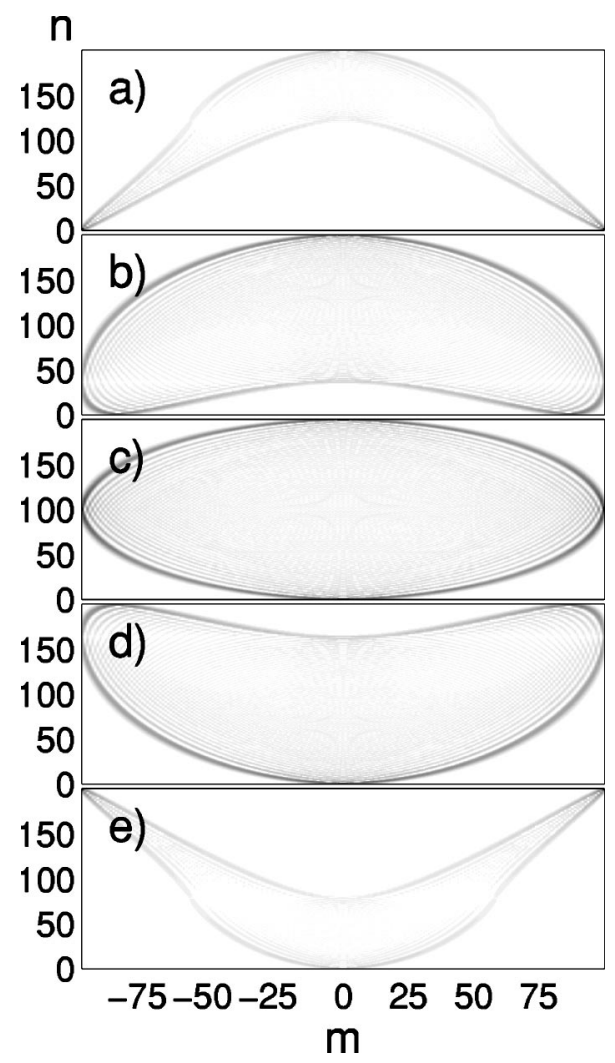

FIG. 2. Probability density for all eigenstates versus magnetic quantum number $m$ for $G=$ (a) -10 , (b) -2 , (c) 0 , (d) 2, and (e) 10 . The lower part of the spectrum is well described by the stationary solutions for the effective potential, Eq. (21). Units are dimensionless. 

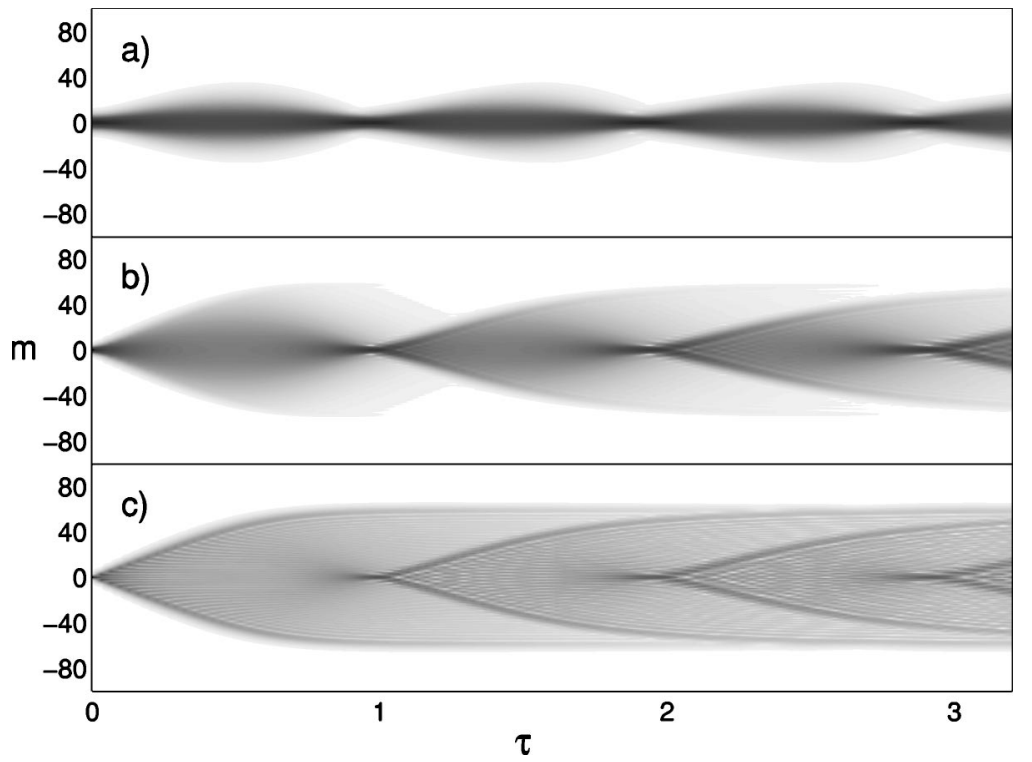

FIG. 3. Probability amplitudes $c_{m}$ as function of the dimensionless time $\tau$ for $G_{\mathrm{dyn}}=10$ and for $G_{\text {in }}=$ (a) 100 , (b) 2000, and (c) 40000 .
The specific example that we consider is the sudden transition for a squeezed state in the Mott regime with $G=G_{\text {in }}$ $\gg 0$ to a new static potential with $G=G_{\text {dyn }}$ that corresponds to a superfluid ground state, both for repulsive and for attractive interactions. In terms of the effective potential (21) this corresponds to taking a squeezed initial state and suddenly widening the potential, and for attractive interaction, introducing a central barrier.

\section{A. Dynamics for $\boldsymbol{G}_{\mathrm{dyn}}>\mathbf{0}$}

Consider first the case of repulsive interactions, $G_{\mathrm{dyn}}>0$. Figure 3 shows the dynamics of an initially squeezed state as a function of time for three different values of $G_{\text {in }}$. The lowest value of $G_{\text {in }}$, shown in Fig. 3(a), results in the harmonic breathing of the amplitudes $c_{m}$ around $x=0$ (or $m=0$ ). Increasing the strength of the interaction, and thus increasing the initial squeezing, alters the dynamics markedly. The larger "momentum" components associated with a narrower initial wave function $c(x, \tau=0)$ allow the atoms to probe regions of $V_{\text {eff }}(x)$ where its anharmonicity becomes more noticeable, as shown in Figs. 3(b) and 3(c).

This behavior is reflected in the fringe visibility (11), which is illustrated in Fig. 4 in both the long- and short-time limits. As expected for a system with an anharmonic spectrum, $V$ exhibits a sequence of collapses and revivals. Figure 5 further shows the short-time oscillations of the visibility as a function of the final energy ratio $G_{\text {dyn }}$. Larger interaction strengths are seen to result in slower oscillations and slower damping. This is further illustrated in Fig. 6, which shows the oscillation period as a function of the final tunneling energy $\Delta E$ for several values of the two-body interaction energy $g$. This period is seen to approach the tunneling time as $g \rightarrow 0$, in qualitative agreement with the experimental results of Ref. [41]. The expectation value of any operator consists of both a time-dependent part and a static part, the latter consisting of terms diagonal in the energy eigenstates
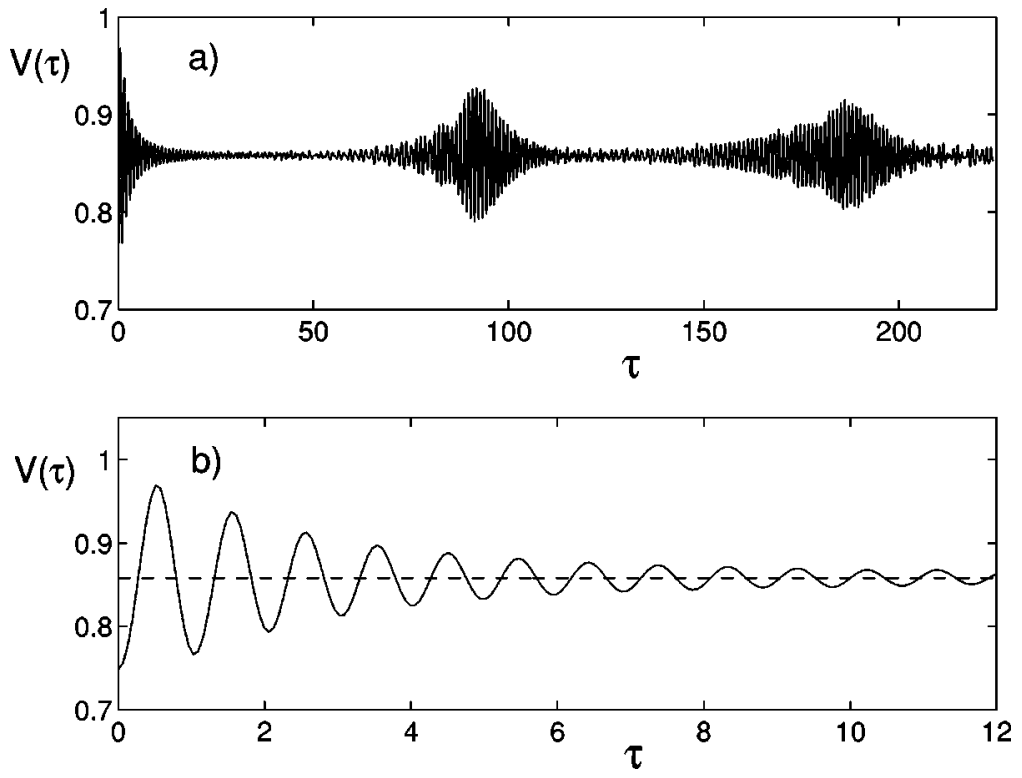

FIG. 4. Visibility $V$ as a function of the dimensionless time $\tau$. (a) shows the long-time collapses and revivals of $V$, while the short-time behavior is shown in more detail in (b). The dashed line shows the background visibility $V_{\mathrm{bg}}$ calculated from Eq. (25). 


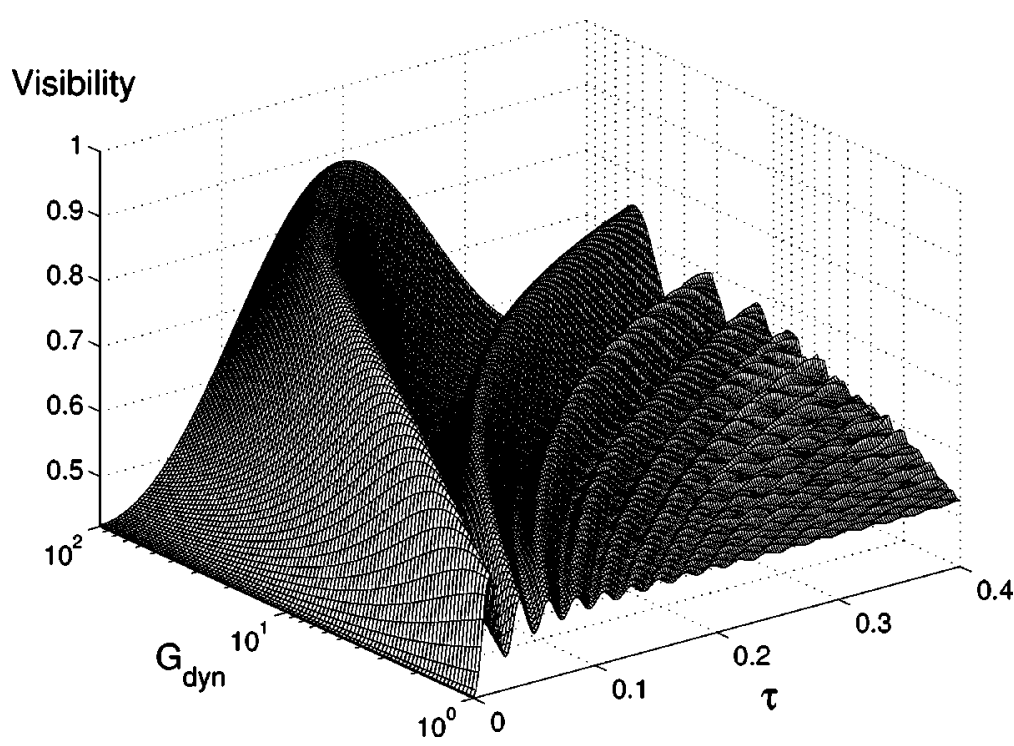

FIG. 5. Visibility $V$ as a function of the dimensionless time $\tau$ and the final energy ratio $G_{\text {dyn }}$ for $N=200$.

as well as contributions from matrix elements from energydegenerate states. The static part of the visibility, which is seen as the background value in Fig. 4, is approached as the oscillations collapse after some time. Using Eq. (11) together with Eqs. (7) and (8), the background contribution to the visibility is found to be

$$
\begin{aligned}
V_{\mathrm{bg}}= & \frac{1}{2 J} \sum_{n=0}^{n_{\max }}\left|D_{n}(0)\right|^{2} \\
& \times\left[\sum_{m=-J+1}^{J} c_{m}^{n} c_{m-1}^{n} \sqrt{(J+m-1)(J-m+2)}\right. \\
& \left.+\sum_{m=-J}^{J-1} c_{m}^{n} c_{m+1}^{n} \sqrt{(J-m-1)(J+m+2)}\right],
\end{aligned}
$$

where the coefficients $c_{m}^{n}$ have been taken to be real without loss of generality since they describe a transformation between real functions. In principle Eq. (25) contains contributions from all combinations in the expectation value that are nonoscillatory. Figure 4(b) illustrates the excellent agreement between this expression and the full dynamical results.

In the continuum limit Eq. (25) reduces to

$$
V_{\mathrm{bg}} \approx \sum_{n=0}^{n_{\max }}\left|d_{n}(0)\right|^{2} \int_{-1}^{1}\left|C_{n}(x)\right|^{2} \sqrt{1-x^{2}} d x,
$$

where the cutoff $n_{\max }$ corresponds to the quantum number of the highest occupied eigenstate. We see from Eq. (26), which is a sum of positive terms, that only those eigenfunctions that are sufficiently well localized around $x=0$ contribute appreciably to $V_{\mathrm{bg}}$.

This sensitivity of the background visibility to the initial state of the atomic sample is illustrated in Fig. 7, which shows $V_{\mathrm{bg}}$ as a function of $G_{\text {in }}$ for several values of $G_{\text {dyn }}$, and

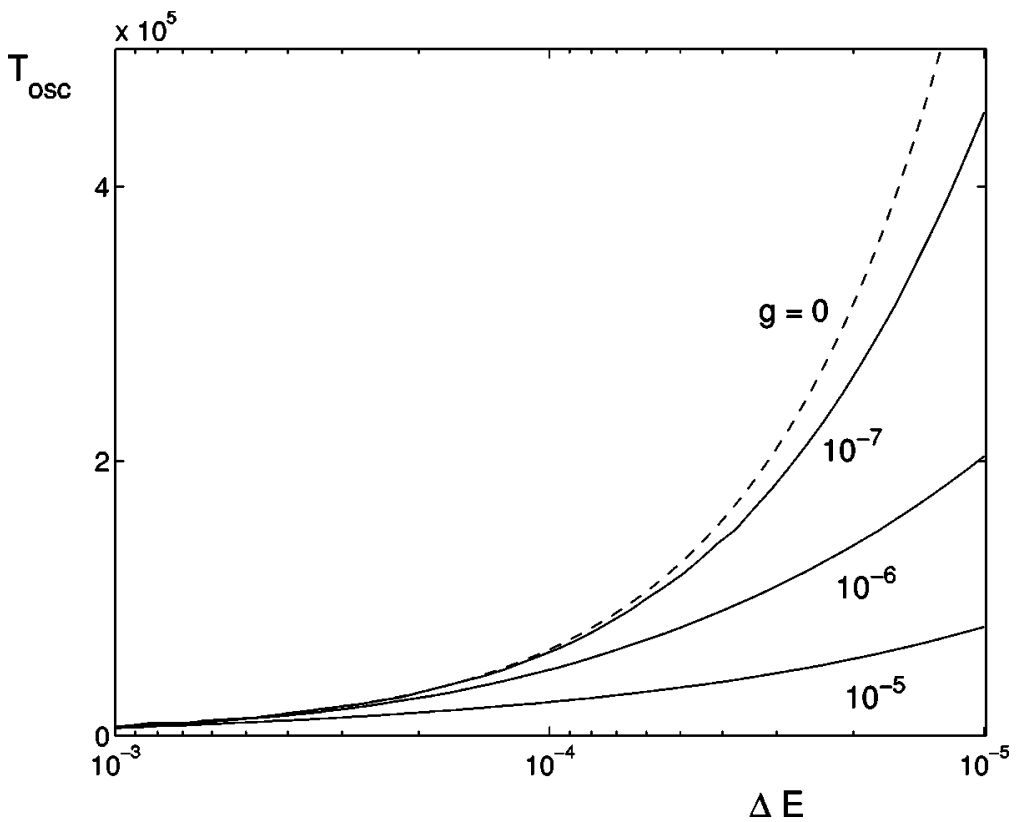

043603-6
FIG. 6. Period of oscillations of $V$, in arbitrary units, as a function of the final tunneling energy $\Delta E$ (arbitrary units) for several values of the twobody interaction energy $g$. 


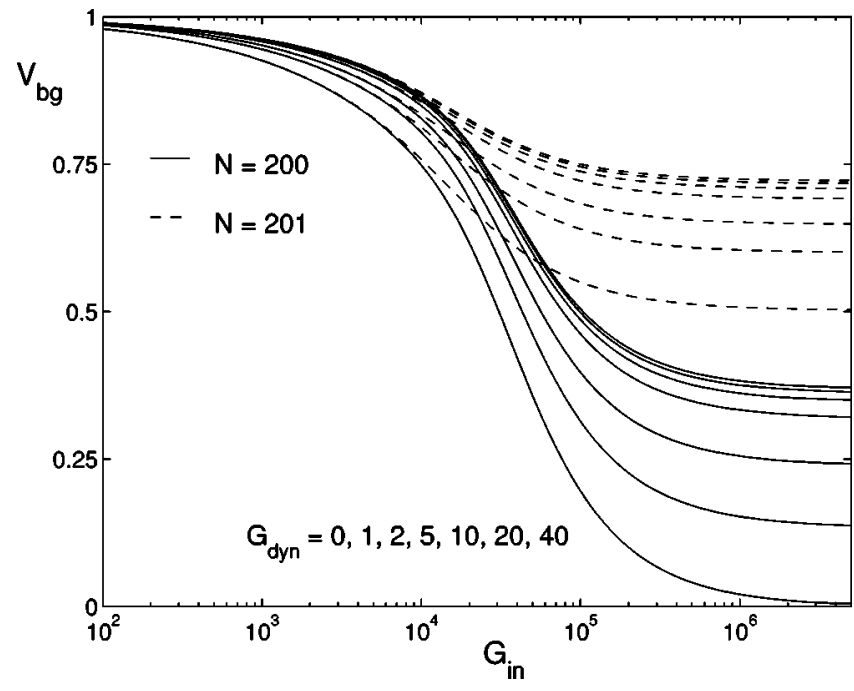

FIG. 7. Background visibility for particle numbers $N=200$ and 201 for several values of final energy ratio $G_{\text {dyn }}$. The final contrast decreases for increasing $G_{\text {dyn }}$.

for $N=200$ and 201 particles. The quantitative difference between odd and even particle numbers, which was already present in the adiabatic regime [5], results from the two-well analog of the situation in optical lattices, where integer filling results in a Mott-insulator transition whereas half-integer filling does not. The dependence of $V_{\mathrm{bg}}$ on the initial state of the atomic field observed in the regime of large $G_{\max }$ can be exploited to determine its degree of squeezing, and in particular to determine the maximal squeezing reached in an experiment of the type of Ref. [4].

To gain further insight into into the dynamics of the system, we now turn to the phase-space representation $Q(\varphi, \theta)$. Figure 8 shows the quasiprobability distribution $Q(\varphi, \theta)$, plotted on the Bloch sphere at four equidistant times. From the Hamiltonian (9), the initial distribution is rotated around the $\hat{J}_{x}$ axis by quantum tunneling, as is already apparent in Fig. 8(b). In addition it is rapidly deformed [see Fig. 8(c)] as

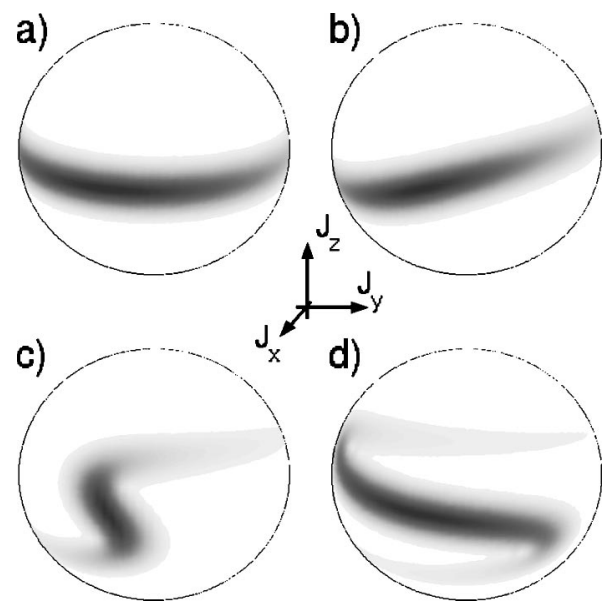

FIG. 8. Snapshots of $Q(\varphi, \theta)$ at the dimensionless times $\tau$ $=[0,0.25,0.5,0.75]$ for $G_{\text {dyn }}=10$. The initial state is the highly squeezed ground-state distribution with $G_{\text {in }}=8000$. The orientation of the Cartesian coordinate system is shown for reference. a result of the twisting induced by two-body collisions. Specifically, the effect of the collisions is to twist $Q(\varphi, \theta)$ around the polar axis, counterclockwise in the northern hemisphere and clockwise in the southern hemisphere. $Q(\varphi, \theta)$ finally returns close to its initial state in Fig. 8(d). The dynamics occurs faster than would be expected from tunneling alone as the interaction makes the flow of $Q(\varphi, \theta)$ take a shortcut that avoids the polar region of the Bloch sphere. This explains intuitively the more rapid period of oscillations of the visibility of Fig. 6 with increasing $g$.

\section{B. Dynamics for $G<0$}

We now turn for completeness to a brief discussion of the atomic dynamics in the regime of attractive two-body interactions, $G_{\text {dyn }}<0$.

Figure 9 shows the evolution of the probability amplitudes $c_{m}(\tau)$ for three values of $G_{\text {dyn }}<0$. For the case (a) of weak attractive interactions the atomic state $|\psi\rangle$ $=\Sigma_{m}(-1)^{m} c(m, t)|J, m\rangle$ exhibits a breathing behavior. We also observe that an increasingly attractive interaction does not force all particles into one or the other potential well. Rather, the dynamics results in a symmetric distribution with on average $\left\langle\hat{a}_{L}^{\dagger} \hat{a}_{L}\right\rangle=\left\langle\hat{a}_{R}^{\dagger} \hat{a}_{R}\right\rangle=N / 2$ atoms in each potential well, far from the ground state of the system.

The range of magnetic moments $m$ (or $x$ ) covered by the atomic state first increases with the interaction strength, as illustrated in Fig. 9(b). This trend persists until all $m$ quantum numbers are encompassed, but further increasing $\left|G_{\text {dyn }}\right|$ past this point prevents the system from reaching $m= \pm J$ through the presence of early reflections. The fact that this behavior cannot be understood in terms of the effective potential $V_{\text {eff }}$ is an indication of the breakdown of the continuous approximation, which permits larger momentum changes than allowed by the discrete original system. In this sense, this situation is the reverse from the familiar problem where a discretized model cannot faithfully reproduce all features of its continuous counterpart.

This behavior can, however, be intuitively understood by examining the dynamics of the quasiprobability distribution $Q(\varphi, \theta)$, as illustrated in Figs. 10 and 11. For sufficiently weak attractive interaction, the distribution $Q(\varphi, \theta)$ rotates nearly rigidly in time around the direction $\hat{J}_{x}$, with some slight distortion near its tips due to the twisting flow. For a strongly squeezed initial state, which corresponds to an initial distribution covering a large fraction of the equatorial region, this rotation leads after some time to the occupation of states up to $m= \pm J$.

The situation is markedly different as $\left|G_{\text {dyn }}\right|$ is increased: in this case, the twisting action of the two-body interactions becomes dominant, and in terms of the Bloch-sphere distribution the flow of $Q(\varphi, \theta)$ results in its wrapping around the pole but never reaching it, as seen in Fig. 11. Note also the destructive interferences in $Q(\varphi, \theta)$ resulting from the superposition of distinct phase-space trajectories.

\section{CONCLUSION}

In this paper, we have analyzed the dynamics of a BoseEinstein condensate of interacting atoms trapped in a double- 


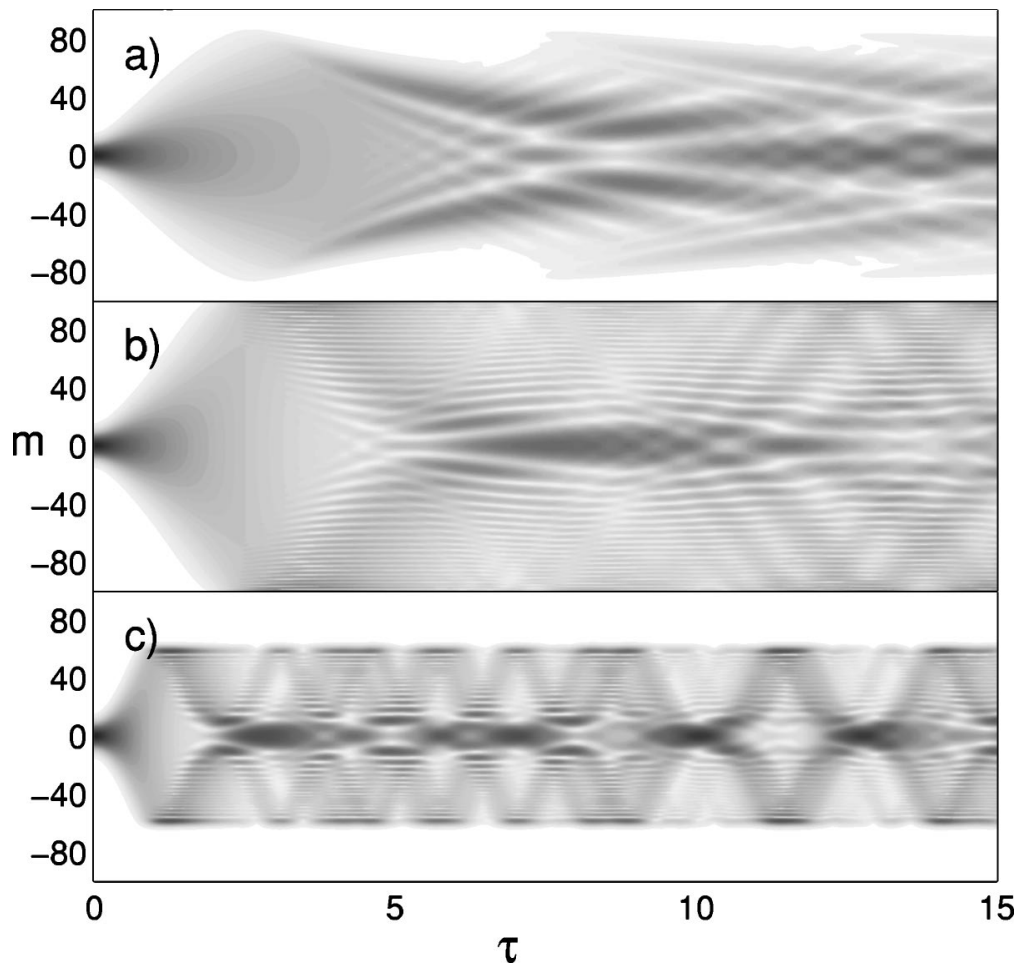

FIG. 9. Probability amplitudes $c_{m}(\tau)$ as a function of the dimensionless time $\tau$ for $G_{\text {in }}=10$ and $G_{\text {dyn }}<0$ : $G_{\text {dyn }}=($ a $)-1$, (b) -2 , and (c) -10 .

well potential whose characteristics are changed suddenly. In addition to its intrinsic interest, this study is motivated by ongoing attempts to develop sub-shot-noise atom interferometry based on the use of nonclassical states of the matterwave field. Double-well and lattice potentials offer an attractive way to achieve a significant number squeezing of the atomic state. Following such a state preparation, Tuchman and Kasevich [42,43] have observed a sharp feature, a socalled "contrast resonance," in the detection of weak perturbations when the potential was suddenly switched to the
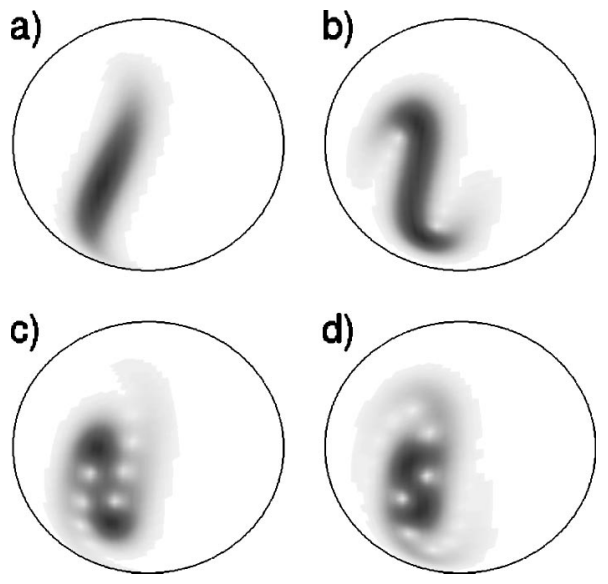

FIG. 10. Four snapshots of $Q(\varphi, \theta)$ taken at equidistant dimensionless times $\tau=2.5,5.0,7.5,10$. Here $G_{\mathrm{dyn}}=-1$. As in Fig. 8, the effects of nonlinearity are evident in the twisting motion of the distribution, although the oscillatory motion is now slowed down rather than accelerated. Same initial state as in Fig. 9. superfluid regime. With the present results at hand, the next step toward a full understanding of this remarkable feature will be to add a position-dependent potential, such as, e.g., a gravitational potential that breaks the symmetry of the double well (or the lattice) and leads to the observed contrast resonance. It is hoped that when extended to that situation, the wave-packet and phase-space analysis developed in the present paper will lead to a detailed understanding of this system and allow a quantitative assessment of its use in subshot-noise matter-wave interferometry.
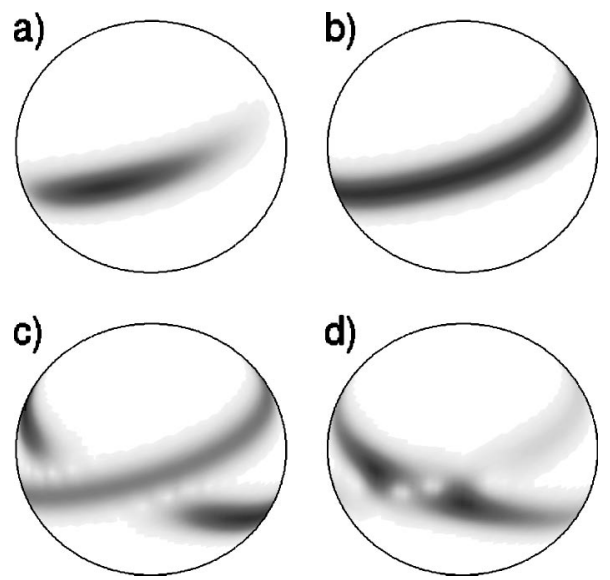

FIG. 11. Four snapshots of $Q(\varphi, \theta)$ taken at equidistant dimensionless times $\tau=0.5,1.0,1.5,2.0$. Here $G_{\mathrm{dyn}}=-10$. In comparison with the dynamics in Fig. 10, the dynamics is seen to be markedly different as the trajectories here wrap around the north pole instead. Same initial state as in Fig. 9. 


\section{ACKNOWLEDGMENTS}

We thank Ari Tuchman and Mark Kasevich for stimulating discussions and for making their results [41-43] available to us prior to publication. This work was supported in part by the U.S. Office of Naval Research, by the National Science Foundation, by the U.S. Army Research Office, by the National Aeronautics and Space Administration, and by the Joint Services Optics Program.
[1] P. Meystre, Atom Optics (Springer, New York, 2001).

[2] M. H. Anderson et al., Science 269, 198 (1995); K. B. Davis et al., Phys. Rev. Lett. 75, 3969 (1995).

[3] D. Jaksch et al., Phys. Rev. Lett. 82, 1975 (1999).

[4] C. Orzel, A. K. Tuchman, M. L. Fenselau, M. Yasuda, and M. A. Kasevich, Science 291, 5512 (2001).

[5] M. Jääskeläinen, W. Zhang, and P. Meystre, Phys. Rev. A 70, 063614 (2004).

[6] J. F. Corney, G. J. Milburn, and W. Zhang, Phys. Rev. A 59, 4630 (1999).

[7] L. Isella and J. Ruostekoski, e-print quant-ph/0409475.

[8] M. P. A. Fisher, P. B. Weichman, G. Grinstein, and D. S. Fisher, Phys. Rev. B 40, 546 (1989).

[9] D. Jaksch, C. Bruder, J. I. Cirac, C. W. Gardiner, and P. Zoller, Phys. Rev. Lett. 81, 3108 (1998).

[10] M. Greiner, O. Mandel, T. Esslinger, T. W. Hänsch, and I. Bloch, Nature (London) 415, 39 (2002).

[11] M. J. Steel and M. J. Collett, Phys. Rev. A 57, 2920 (1998).

[12] J. Javanainen and M. Yu. Ivanov, Phys. Rev. A 60, 2351 (1999).

[13] S. Raghavan, A. Smerzi, S. Fantoni, and S. R. Shenoy, Phys. Rev. A 59, 620 (1999).

[14] M. Albiez et al., e-print cond-mat/0411757.

[15] R. W. Robinett, Phys. Rep. 392, 1 (2004).

[16] G. J. Milburn, J. Corney, E. M. Wright, and D. F. Walls, Phys. Rev. A 55, 4318 (1997).

[17] A. Polkovnikov, Phys. Rev. A 68, 033609 (2003).

[18] J. A. Dunningham and K. Burnett, Phys. Rev. A 70, 033601 (2004)

[19] G. Kalosakas and A. R. Bishop, Phys. Rev. A 65, 043616 (2002).

[20] G. Kalosakas, A. R. Bishop, and V. M. Kenkre, Phys. Rev. A 68, 023602 (2003).

[21] P. Buonsante, R. Fransozi, and V. Penna, J. Phys. B 37, S195 (2004).

[22] J. Plata, Phys. Rev. A 69, 033604 (2004).

[23] O. Morsch, J. H. Müller, D. Camini, M. Cristiani, P. B. Blakie, C. J. Williams, P. S. Julienne, and E. Arimondo, Phys. Rev. A 67, 031603(R) (2003).

[24] A. Polkovnikov, S. Sachdev, and S. M. Girvin, Phys. Rev. A
66, 053607 (2002).

[25] E. Altman and A. Auerbach, Phys. Rev. Lett. 89, 250404 (2002).

[26] A.-M. Rey, B. L. Hu, E. Calzetta, A. Roura, and C. W. Clark, Phys. Rev. A 69, 033610 (2004).

[27] P. A. Ruprecht, M. J. Holland, K. Burnett, and M. Edwards, Phys. Rev. A 51, 4704 (1995); G. Baym and C. J. Pethick, Phys. Rev. Lett. 76, 6 (1996); Y. Kagan, G. V. Shlyapnikov, and J. T. M. Walraven, ibid. 76, 2670 (1996).

[28] F. Kh. Abdullaev, A. Gammal, L. Tomio, and T. Frederico, Phys. Rev. A 63, 043604 (2001).

[29] A. Gammal, L. Tomio, and T. Frederico, Phys. Rev. A 66, 043619 (2002); C. Huepe, L. S. Tuckerman, S. Metens, and M. E. Brachet, ibid. 68, 023609 (2003); A. G. de Sousa, A. B. F. da Silva, G. C. Marques, and V. S. Bagnato, ibid. 70, 063608 (2004).

[30] E. Sakellari, N. P. Proukakis, and C. S. Adams, J. Phys. B 37, 3681 (2004).

[31] J. F. Corney, G. J. Milburn, and W. Zhang, Phys. Rev. A 59, 4630 (1999).

[32] M. Kitagawa and M. Ueda, Phys. Rev. A 47, 5138 (1993).

[33] Y. Wu and X. Yang, Phys. Rev. A 68, 013608 (2003).

[34] D. T. Pegg and S. M. Barnett, Phys. Rev. A 39, 1665 (1989); A. Luis and L. L. Sanchez-Soto, ibid. 48, 4702 (1993).

[35] J. Yeazell and U. Turgay, The Physics and Chemistry of Wave Packets (Wiley, New York, 2000).

[36] W. P. Schleich, Quantum Optics in Phase Space (Wiley-VCH, Berlin, 2001).

[37] F. T. Arecchi, E. Courtens, R. Gilmore, and H. Thomas, Phys. Rev. A 6, 2211 (1972).

[38] S. Sachdev, Quantum Phase Transitions (Cambridge University Press, Cambridge, U. K., 1999).

[39] O. Atabek, R. Lefebvre, M. Garcia Sucre, J. Gomez-Llorente, and H. Taylor, Int. J. Quantum Chem. 15, 211 (1991).

[40] M. Jääskeläinen and S. Stenholm, Phys. Rev. A 68, 033607 (2003).

[41] A. K. Tuchman, C. Orzel, M. L. Fenselau, A. Povkolnokov, and M. Kasevich (unpublished).

[42] A. K. Tuchman and M. Kasevich (unpublished).

[43] A. K. Tuchman, Ph.D. thesis, Yale University, 2004. 